\title{
Sensitivity Analysis of User Equilibrium Flows Revisited
}

\author{
Byung Do Chung • Hsun-Jung Cho • Terry L. Friesz • \\ Henh Huang • Tao Yao
}

Published online: 26 November 2013

(C) Springer Science+Business Media New York 2013

\begin{abstract}
This paper addresses certain misconceptions regarding what is known and what may be expected when performing sensitivity analyses of network user equilibrium flow patterns. Our presentation relies on a simple observation: any given user equilibrium sensitivity analysis technique should be employed only when the regularity conditions on which it is based are satisfied. Violating regularity, as we show through previously published numerical examples, as well as new examples presented here for the first time, may well lead to incorrect results when the Tobin-Friesz sensitivity analysis method is applied. This is especially so when the most critical regularity assumption of the TobinFriesz method, namely that the unperturbed solution must be a nondegenerate extreme point, is violated. We also illustrate how a degenerate unperturbed solution may sometimes be modified to obtain an appropriate nondegenerate solution, thereby allowing the Tobin-Friesz method to be applied.
\end{abstract}

Keywords Sensitivity analysis $\cdot$ Network user equilibrium $\cdot$ Regularity condition

B. D. Chung

Department of Systems Management Engineering, Sungkyunkwan University, Suwon, South Korea e-mail: bd.chung@skku.edu

H.-J. Cho $\cdot$ H. Huang

Department of Transportation Technology \& Management, National Chiao Tung University, Hsinchu, Taiwan

H.-J. Cho

e-mail: hjcho001@gmail.com

T. L. Friesz $\cdot$ T. Yao $(\triangle)$

Department of Industrial and Manufacturing Engineering, The Pennsylvania State University, University Park, PA, USA

e-mail: taoyao@psu.edu

T. L. Friesz

e-mail: tfriesz@psu.edu 


\section{Introduction}

The type of sensitivity analysis we consider in this paper is based on the simultaneous perturbation of parameters in a static user equilibrium model. We assume herein that the reader is aware of the importance of being able to easily approximate a new equilibrium following such a perturbation. The work by Fiacco (1983), Tobin (1986), Kyparisis (1987) and Dafermos (1988) on the sensitivity analysis of nonlinear programs and abstract variational inequalities eventually inspired several efforts to create a method for the sensitivity analysis of user equilibrium.

The history of user equilibrium sensitivity analysis is interesting, and, for our purposes herein, may be considered to begin with Tobin and Friesz (1988). In the Tobin-Friesz approach to user equilibrium sensitivity analysis, an unperturbed nondegenerate extreme point must be known from the outset in order to initiate sensitivity analysis calculations. The formulae for sensitivity analysis obtained under the nondegeneracy assumption accord with intuition, and are easy to derive and understand. Various other user equilibrium sensitivity analysis methods including Qiu and Magnanti (1989), Yang (1997), Cho et al. (2000), Patriksson and Rockafellar (2003), Patriksson (2004), Yang and Bell (2007) and Lu (2008) have been developed since Tobin and Friesz (1988). Sensitivity analysis methods have been effectively used for various transportation applications, including but not limited to network design (Friesz et al. (1990) and Davis (1994)), origin-destination matrix estimation (Yang et al. (2001)), and optimal pricing (Ying and Yang (2005)).

This paper has been written to clarify the regularity conditions governing application of the Tobin-Friesz method (TFM) for user equilibrium sensitivity analysis presented in Tobin and Friesz (1988) and rederived in Cho et al. (2000). We have found that certain statements and numerical examples found in Patriksson (2004), Josefsson and Patriksson (2007) and Marcotte and Patriksson (2007), if taken out of context, leave the impression that the TFM for user equilibrium sensitivity analysis is somehow "wrong" when, in fact, it works quite well provided its application is limited to those problems fulfilling the regularity conditions reviewed in this paper.

We wish to make clear from the outset that the sensitivity analysis formulae developed by Josefsson and Patriksson (2007), Marcotte and Patriksson (2007) and $\mathrm{Lu}$ (2008) are more general in some aspects than the TFM. We note that a central theorem in Patriksson (2004) is unproven since the proof is incorrect as shown in Robinson (2006). History, however, plays a significant role in the scholarly conversation about sensitivity analysis of user equilibrium flows. In particular, the TFM was developed some 15 to 20 years prior to the alternative literature cited above, at a time when there was considerable doubt about whether sensitivity analysis of variational inequalities based on path variables was possible. It is only natural that, as the first method for user equilibrium sensitivity analysis, the TFM involves more restrictive regularity conditions than those of subsequently published works.

The aforementioned lack of generality notwithstanding, the TFM is a self-consistent user equilibrium sensitivity analysis technique. As such, the TFM does not produce erroneous results when the user equilibrium to which it is applied fulfills the assumptions used for derivation of the TFM presented in this paper. Although there are now more general methods for user equilibrium sensitivity analysis, such generality comes at the price of increased mathematical sophistication that may place alternative methods 
beyond the comprehension of some practitioners. In mathematics, generality is always thought to be a virtue. However, in operations research, engineering and the social sciences, users of mathematical tools vote with their feet; that is, the most general mathematical tools may fail to be the most popular mathematical tools. Such is the nature of scholarly inquiry and the march of knowledge.

\section{The Principal Issues}

Although the TFM for sensitivity analyses, which was re-derived using alternative arguments by Cho et al. (2000), has been widely used, it has been criticized by Patriksson (2004), Josefsson and Patriksson (2007), Marcotte and Patriksson (2007), Yang and Bell (2007) and Yang and Huang (2005) on the basis of the following:

1. A non-standard definition of user equilibrium is employed in Tobin and Friesz (1988). We agree that the definition of user equilibrium employed is not general, and it represents a limitation of the analysis presented in Tobin and Friesz (1988). However, as we show in this paper, the results derived in Tobin and Friesz (1988) do not depend on using that nonstandard definition and may be derived using the traditional definition of user equilibrium.

2. A non-standard definition of strict complementary slackness is employed in Tobin and Friesz (1988). We agree that the definition of strict complementarity used in Tobin and Friesz (1988) is nonstandard. However, we show in this paper that the TFM may be derived using the traditional definition of strict complementary slackness.

3. To apply the TFM, one must begin with an unperturbed solution that is a nondegenerate extreme point. This regularity condition represents a critical assumption; it is the key reason why the TFM is easy to derive. Example problems have been published that show the TFM may fail when the nondegeneracy assumption is relaxed. However, it is possible to modify the degenerate solutions employed in example 7.3.2 of Josefsson and Patriksson (2007), as shown in Section 4.1 .5 of this paper, and example 8 of Yang and Bell (2007), as shown in Section 4.2.3 of this paper, to create valid initial solutions that are nondegenerate. However, our remarks should not be misconstrued as a claim that such modifications will always be possible, for they will not. However, in the event it is possible to construct a nondegenerate extreme point solution from a degenerate solution, the TFM works. Moreover, the rederived method by Cho et al. (2000) can sometimes be applied to cases where an unperturbed solution is a nondegenerate extreme point as shown in Section 4.1.6 of this paper for examples 7.3.2 and 7.3.3 of Josefsson and Patriksson (2007) and Section 4.2.4 of this paper for example 8 of Yang and Bell (2007).

4. From Tobin and Friesz (1988), it is clear that existence and invertibility of the Jacobian matrix of the path cost vector, a submatrix of the entire Jacobian matrix formed from the Kuhn-Tucker conditions (including the complementary slackness conditions), are crucial to the validity of the 
TFM. Differentiability of the relevant functions assures existence of that Jacobian, and the stipulation of differentiability as a regularity condition is not at issue. However, Bell and Iida (1997) correctly observed that the Jacobian of the path cost vector is not invertible when the number of paths is larger than the number of arcs, as example 6 of Yang and Bell (2007) illustrates. In Section 4.2.1 of this paper, we show that sometimes it is possible for the whole Jacobian matrix to be invertible even though the aforementioned submatrix (the Jacobian matrix of the path cost vector) is not invertible. Moreover, in assessing the TFM, one should not forget that the alternative derivation of identical sensitivity analysis formulae in Cho et al. (2000) was performed to overcome the potential noninvertibility of the Jacobian matrix of the path cost vector, provided the appropriate derivatives needed to express the Jacobian may be calculated.

5. Violation of traditional strict complementarity may occur. Because one of the regularity conditions stipulated by Tobin and Friesz (1988) is strict complementarity, it is reasonable to expect that the violation of some form of strict complementarity would generally prevent sensitivity analysis based on the TFM. However, in this paper, we show by numerical example that sensitivity analysis may sometimes be performed using the TFM even if strict complementarity is violated, provided there is differentiability at the unperturbed user equilibrium solution. Example 5 in Patriksson (2004) is an instance wherein both strict complementarity and differentiability fail to hold. Per se, sensitivity analysis of such a non-differentiable problem cannot be conducted using the TFM. However, when we consider example 7.3.1 of Josefsson and Patriksson (2007), which does not fulfill strict complementarity but meets the differentiability standard, we find the TFM may be used successfully, as we illustrate in Section 4.1.2

\section{User Equilibrium Sensitivity Analysis}

We will employ the specific notation presented in Table 1. We assume the user equilibrium problem possess $s$ parameters that are subject to perturbation; these form the vector $\varepsilon \in \mathfrak{R}^{s}$. The reader should note that the number $s$ of perturbed parameters has no a priori relationship to the number of decision variables, the number of equality constraints or the number of inequality constraints found in a given user equilibrium problem.

To represent the perturbation of a given user equilibrium problem, we explicitly express the dependence of the impacted arc and path flows, cost functions and demands on the perturbation vector $\varepsilon \in \mathfrak{R}^{s}$. That is, we will write

$$
\begin{aligned}
& h(\varepsilon) \\
& C(h(\varepsilon), \varepsilon)
\end{aligned}
$$

to denote the vector of perturbed path flows and the vector of perturbed path costs, respectively. Similar notation of course applies to other perturbed entities. We will not elaborate further on such notation, since it is intuitive and widely used in the sensitivity analysis literature. Using the notation of Table 1, 
Table 1 The notation

\begin{tabular}{ll}
\hline Notation & Description \\
\hline$N$ & the set of nodes of the network \\
nodes in the network & the set of arcs of the network \\
an arc of the network, $a=(i, j)$ & the set of origin-destination pairs \\
$A$ & an origin-destination pair, $w=(i, j)$ \\
$W \in A$ & the set of paths between origin-destination pair \\
$w \in W$ & the arc-path incidence matrix, where $\Delta_{a p}=1$ if arc $a$ is in path $p, 0$ otherwise \\
$P_{w}$ & the origin-destination/path incidence matrix, where $\Lambda_{w p}=1$ if path $p \in P_{w}, 0$ otherwise \\
$\Delta=\left[\Delta_{a p}\right]$ & traffic required between origin-destination pair $w$ \\
$\Lambda=\left[\Lambda_{w p}\right]$ & the vector of all traffic requirements \\
$Q_{w}$ & the flow on path $p$ \\
$Q=\left[Q_{w}: w \in W\right]$ & the vector of all path flows \\
$h_{p}$ & the flow on arc $a$ \\
$h=\left[h_{p}: p \in P\right]$ & the vector of all arc flows, note that $v=\Delta h$ \\
$v_{a}$ & the unit cost on arc $a$ as a function of all arc flows \\
$v=\left[v_{a}: a \in A\right]$ & the vector of arc unit cost functions \\
$c_{a}(v)$ & the unit cost on path $p$ as a function of all path flows \\
$c(v)=\left[c_{a}(v): a \in A\right]$ & the vector of path unit cost function \\
$C_{p}(h)$ & path unit costs are the sum of arc unit costs \\
$C(h)=\left[C_{p}(h): p \in P\right]$ & minimum unit path cost for origin-destination pair $w \in W$ \\
$C(h)=\Delta^{T} c(v)=\Delta^{T} c(\Delta h)$ & the vector of minimum travel costs \\
$\lambda_{w}$ & \\
$\lambda=[\lambda: w \in W]$ & \\
\hline &
\end{tabular}

together with the perturbation notation convention just mentioned, the unperturbed user equilibrium problem of interest is:

$$
\begin{gathered}
g_{p}\left(h^{*}(0)\right) \equiv-h_{p}^{*}(0)<0, p \in P_{w} \Rightarrow C_{p}\left(h^{*}(0), 0\right)=\lambda_{w} \forall w \in W \\
C_{p}\left(h^{*}(0), 0\right)-\lambda_{w} \geq 0 \quad \forall w \in W, p \in P_{w} \\
d_{w}\left(h^{*}(0), 0\right) \equiv \sum_{p \in P_{w}} h_{p}^{*}(0)-Q_{w}=0 \forall w \in W
\end{gathered}
$$

Let us define the set of feasible solutions

$$
\Omega_{h}(0)=\left\{h(0): d_{w}(h(0), 0)=0, g_{p}(h(0)) \leq 0\right\}
$$

It is not difficult to show that Eqs. (1-3) may be equivalently stated as the variational inequality

$$
\begin{aligned}
& V I_{h}(0) \text { : find } h^{*}(0) \in \Omega_{h}(0) \text { such that } \\
& \quad\left[C\left(h^{*}(0), 0\right)\right]^{T}\left(h(0)-h^{*}(0)\right) \geq 0 \quad \forall h(0) \in \Omega_{h}(0)
\end{aligned}
$$


since we take demand to be inelastic. The variational inequality (5), under the assumption that path costs are additive in arc costs, is equivalent to

where

$$
\begin{aligned}
& V I_{v}(0): \text { find } v^{*}(0) \in \Omega_{v}(0) \text { such that } \\
& \quad\left[c\left(v^{*}(0), 0\right)\right]^{T}\left(v(0)-v^{*}(0)\right) \geq 0 \quad \forall v(0) \in \Omega_{v}(0)
\end{aligned}
$$

$$
\Omega_{v}(0)=\left\{v(0): d_{w}(h(0), 0)=0, g_{p}(h(0)) \leq 0, v(0)=\Delta h(0)\right\}
$$

is the set of feasible arc flows.

Clearly, the perturbed version of Eqs. (5-6) are

$$
\begin{aligned}
& V I_{h}(\varepsilon): \text { find } h^{*}(\varepsilon) \in \Omega_{h}(\varepsilon) \text { such that } \\
& \quad\left[C\left(h^{*}(\varepsilon), \varepsilon\right)\right]^{T}\left(h(\varepsilon)-h^{*}(\varepsilon)\right) \geq 0 \quad \forall h(\varepsilon) \in \Omega_{h}(\varepsilon) \\
& V I_{v}(\varepsilon): \text { find } v^{*}(\varepsilon) \in \Omega_{v}(\varepsilon) \text { such that } \\
& \quad\left[c\left(v^{*}(\varepsilon), \varepsilon\right)\right]^{T}\left(v(\varepsilon)-v^{*}(\varepsilon)\right) \geq 0 \quad \forall v(\varepsilon) \in \Omega_{v}(\varepsilon)
\end{aligned}
$$

where we employ the obvious definitions of perturbed feasible sets, namely

$$
\begin{gathered}
\Omega_{h}(\varepsilon)=\left\{h(\varepsilon): d_{w}(h(\varepsilon), \varepsilon)=0, g_{p}(h(\varepsilon)) \leq 0\right\} \\
\Omega_{v}(\varepsilon)=\left\{v(\varepsilon): d_{w}(h(\varepsilon), \varepsilon)=0, g_{p}(h(\varepsilon)) \leq 0, v(\varepsilon)=\Delta h(\varepsilon)\right\}
\end{gathered}
$$

\subsection{Regularity Conditions}

We now introduce the following regularity conditions which are assumed to be in force unless otherwise stated:

Condition 1 Continuous differentiability. In the perturbed equilibrium network flow problem, $c(v, \varepsilon)$ is once continuously differentiable in $(v, \varepsilon)$, and $Q(\varepsilon)$ is once continuously differentiable in $\varepsilon$.

Condition 2 Strong monotonicity. The arc cost vector $c(v, \varepsilon)$ is strongly monotone in $v$ for all $\varepsilon$. As a consequence, the arc flow solution is unique.

Condition 3 Traditional strict complementarity. If $h_{p}^{*}(0)=0$, then $C_{p}^{*}\left(h^{*}(0)\right.$, $0)>\lambda_{w}(0)$ for origin-destination (OD) pair $w \in W$.

\subsection{The Key Formulae}

Now suppose $v^{*}(0)$ to be a locally unique solution to $V I_{v}(0)$. Additionally take $\nabla_{h} g_{p}\left(h^{*}, 0\right)$, for all $p$ such that $g_{p}\left(h^{*}, 0\right)=0$, and $\nabla_{h} d_{w}\left(h^{*}, 0\right)$, for all $w \in W$, to be linearly independent, where the gradients are with respect to path flows. Furthermore, assume that the strict complementary slackness condition

$$
\pi_{p}^{*}(0)>0 \text { when } g_{p}\left(h^{*}(0), 0\right)=0
$$

holds. Then the equilibrium dual variables for inequality constraints and equality constraints, $\pi^{*}(0)$ and $\mu^{*}(0)$ respectively, are unique. Moreover, in a 
neighborhood of $\varepsilon=0$, there exists a unique once continuously differentiable function

$$
y(\varepsilon) \equiv\left[\begin{array}{c}
v^{*}(\varepsilon) \\
\pi^{*}(\varepsilon) \\
\mu^{*}(\varepsilon)
\end{array}\right]
$$

where $v^{*}(\varepsilon)$ is the locally unique arc flow solution to $V I_{v}(\varepsilon)$ assumed previously to be at our disposal, and $\pi^{*}(\varepsilon)$ and $\mu^{*}(\varepsilon)$ are its unique associated multipliers.

Note that $V I_{h}(0)$ may be stated as

$$
\min Z(h(0))=\left[C\left(h^{*}(0), 0\right)\right]^{T} h(0) \quad \text { s.t.h }(0) \in \Omega_{h}(0)
$$

and

$$
\frac{\partial Z(0)}{\partial h_{p}}=\sum_{a \in A} \frac{\partial f_{a} \partial Z(0)}{\partial h_{p} \partial f_{a}}=\sum_{a \in A} \delta_{a p} c_{a}(f(0), 0)=C_{p}(h(0), 0)
$$

Thus

$$
\nabla_{h} Z(0)=C\left(h^{*}(0), 0\right)
$$

The derivative of the solution vector $y(\varepsilon)$ with respect to $\varepsilon$ obeys the following form of the implicit function theorem:

$$
\nabla_{\varepsilon} y(0)=\left[\begin{array}{c}
\nabla_{\varepsilon} v^{*}(0) \\
\nabla_{\varepsilon} \pi^{*}(0) \\
\nabla_{\varepsilon} \mu^{*}(0)
\end{array}\right]=\left[J_{y}^{*}(0)\right]^{-1} \cdot\left[J_{\varepsilon}^{*}(0)\right]
$$

where $J_{y}^{*}(0)$ is the Jacobian matrix with respect to y of the following Kuhn-Tucker system for $V I_{h}(0)$ :

$$
\begin{gathered}
C\left(h^{*}(0), 0\right)+\sum_{i=1}^{m} \pi_{i} \nabla_{h} g_{i}\left(h^{*}(0), 0\right)+\sum_{i=1}^{p} \mu_{i} \nabla_{h} d_{i}\left(h^{*}(0), 0\right)=0 \\
\pi_{i} g_{i}\left(h^{*}(0), 0\right)=0 \quad \pi_{i} \geq 0 \text { for } i=1, \ldots, m \\
d_{i}\left(h^{*}(0), 0\right)=0 \text { for } i=1, \ldots, p
\end{gathered}
$$

and $J_{\varepsilon}(0)$ the Jacobian with respect to $\varepsilon$. Result Eq. (12) would seem to provide an immediate opportunity to conduct sensitivity analysis. Unfortunately, as is now widely understood, the gradients in Eq. (13) do not generally exist since path flows are generally not unique. Therefore, Eq. (12) cannot be used as it is presently stated.

To avoid complications arising from the non-uniqueness of path flows, Tobin and Friesz (1988) assumed the unperturbed equilibrium path flow solution on which to base sensitivity analysis corresponds to a nondegenerate 
extreme point of $\Omega_{h}(0)$. More precisely, they invoked the following regularity condition:

Condition 4 Non-degeneracy assumption. There exists $h^{*}(0)$ that is a nondegenerate extreme point of

$$
\Omega_{h}(0)=\left\{h: \Delta h=v^{*}(0), \Lambda h=Q(0), h \geq 0\right\}
$$

in the sense that $h^{*}(0)$ corresponds to a unique basis and the number of paths with positive flow is equal to the rank of $\left[\Delta^{T}, \Lambda^{T}\right]$ after restricting the network to arcs with positive flows.

We know that the perturbed arc flow solution $f^{*}(\varepsilon)$ is continuous with respect to the perturbation vector $\varepsilon$ because of Condition 2 . Thus, provided $\varepsilon$ is small enough, we are assured that those arcs of the perturbed solution with positive flow are the same as those of the unperturbed solution. We are then free to consider only those paths of the nondegenerate extreme point that traverse arcs with positive flow and themselves have positive path flows. We shall refer to the vector of unperturbed, positive, equilibrium path flows found in the manner just described as $h^{0}(0)$. Moreover, because of continuity, we may be sure, for sufficiently small $\varepsilon$, that $-h^{0}(\varepsilon)<0$. As a consequence, the associated dual variables are all zero; that is, $\pi^{0}(0)=\pi^{0}(\varepsilon)=0$.

We shall employ the notation $\Delta^{0}$ for the arc-path incidence matrix corresponding to $h^{0}(0)$. Additionally, we shall use the notation $\Lambda^{0}$ for the OD-path incidence matrix corresponding to $h^{0}(0)$. The matrices $\Delta^{0}$ and $\Lambda^{0}$ also apply to the perturbed solution $h^{0}(\varepsilon)$ by virtue of continuity and the way in which $h^{0}(0)$ has been constructed.

Thus, the key information on which sensitivity analysis is predicated is $h^{0}(0)$, which in turn is predicated on finding a non-degenerate extreme point of $\Omega_{h}(0)$. Tobin and Friesz (1988) suggest searching for a non-degenerate extreme point by varying $b \in \mathfrak{R}^{|h|}$ and repeatedly solving the linear program:

$$
\min b^{T} h \text { s.t. } h \in \Omega_{h}(0)
$$

Other schemes may also be employed, as we shall illustrate later via numerical examples.

As long as the Conditions 1 through 4 mentioned above are satisfied, the derivative of the equilibrium solution vector with respect to $\varepsilon$ may be calculated using the TFM. Let us briefly review what has been said above by placing the essential actions in the order in which they would be executed in an actual application:

1. If Conditions 1 through 3 are satisfied, restrict the network to those arcs with positive arc flows.

2. If possible, determine a nondegenerate extreme point $h^{*}(0) \in \Omega_{h}(0)$ so that Condition 4 is satisfied. Construct $h^{0}(0)$ by considering only those paths of the nondegenerate extreme point $h^{*}(0)$ that traverse arcs with positive flow and themselves have positive path flows.

3. Construct the origin-destination/path incidence matrix $\Lambda^{0}$ and arc-path incidence matrix $\Delta^{0}$ corresponding to $h^{0}(0)$.

4. Create formulae for sensitivity analysis using the implicit function theorem identity (12) adapted to the restricted network created via actions 1, 2 and 3 above, 
recognizing that $\pi^{0}=0$, since all path flows of both the perturbed and unperturbed versions of the restricted network are positive. The relevant result is

$$
\left[\begin{array}{c}
\nabla_{\varepsilon} h^{0}(0) \\
\nabla_{\varepsilon} \mu^{0}(0)
\end{array}\right]=\left[J_{h^{0} \mu^{0}}^{*}(0)\right]^{-1}\left[J_{\varepsilon}^{*}(0)\right]
$$

and

$$
\nabla_{\varepsilon} v^{0}(0)=\Delta^{0} \nabla_{\varepsilon} h^{0}(0)
$$

where $J_{h^{0} \mu^{0}}^{*}(0)$ is the Jacobian of the Kuhn-Tucker conditions for the restricted network with respect to $h^{0}(0)$ and $\mu^{0}(0)$ when $\varepsilon=0$; and $J_{\varepsilon}^{*}(0)$ is the Jacobian of the Kuhn-Tucker conditions for the restricted network with respect to perturbations when $\varepsilon=0$. The Jacobian matrices $J_{h^{0} \mu^{0}}^{*}(0)$ and $J_{\varepsilon}^{*}(0)$ are

$$
\begin{gathered}
{\left[J_{\varepsilon}^{*}(0)\right]=\left[\begin{array}{l}
-\nabla_{\varepsilon} C^{0}\left(h^{*}(0), 0\right) \\
\nabla_{\varepsilon} Q(0)
\end{array}\right]} \\
{\left[J_{h^{0} \mu^{0}}^{*}(0)\right]=\left[\begin{array}{cc}
\nabla_{h} C^{0}\left(h^{*}, 0\right) & -\Lambda^{0 T} \\
\Lambda^{0} & 0
\end{array}\right]}
\end{gathered}
$$

Suppose

$$
\left[J_{h^{0} \mu^{0}}^{*}(0)\right]^{-1}=\left[\begin{array}{ll}
B_{11} & B_{12} \\
B_{21} & B_{22}
\end{array}\right]
$$

then the derivatives with respect to $\varepsilon$ when $\varepsilon=0$ are

$$
\begin{aligned}
& \nabla_{\varepsilon} h^{0}(0)=-B_{11} \nabla_{\varepsilon} C^{0}\left(h^{*}(0), 0\right)+B_{12} \nabla_{\varepsilon} Q(0) \\
& \nabla_{\varepsilon} \mu^{0}(0)=-B_{21} \nabla_{\varepsilon} C^{0}\left(h^{*}(0), 0\right)+B_{22} \nabla_{\varepsilon} Q(0)
\end{aligned}
$$

where

$$
\begin{gathered}
B_{11}=\nabla_{h} C^{0}\left(h^{*}, 0\right)^{-1}\left[I-\Lambda^{0 T}\left[\Lambda^{0} \nabla_{h} C^{0}\left(h^{*}, 0\right)^{-1} \Lambda^{0 T}\right]^{-1} \Lambda^{0} \nabla_{h} C^{0}\left(h^{*}, 0\right)^{-1}\right] \\
B_{12}=\nabla_{h} C^{0}\left(h^{*}, 0\right)^{-1} \Lambda^{0 T}\left[\Lambda^{0} \nabla_{h} C^{0}\left(h^{*}, 0\right)^{-1} \Lambda^{0 T}\right]^{-1} \\
B_{21}=-\left[\Lambda^{0} \nabla_{h} C^{0}\left(h^{*}, 0\right)^{-1} \Lambda^{0 T}\right]^{-1} \Lambda^{0} \nabla_{h} C^{0}\left(h^{*}, 0\right)^{-1} \\
B_{22}=\left[\Lambda^{0} \nabla_{h} C^{0}\left(h^{*}, 0\right)^{-1} \Lambda^{0 T}\right]^{-1}
\end{gathered}
$$

are found using a minimum norm projection to express a mathematical program whose unique solution provides the gradient information needed for sensitivity analysis. 
5. Perform the desired sensitivity analysis according to the following local linear approximation:

$$
\left[\begin{array}{c}
h^{0}(\varepsilon) \\
\mu^{0}(\varepsilon)
\end{array}\right] \approx\left[\begin{array}{c}
h^{0}(0) \\
\mu^{0}(0)
\end{array}\right]+\left[\begin{array}{ll}
\left(\nabla_{\varepsilon} h^{0}(0)\right)^{T} & \left(\nabla_{\varepsilon} \mu^{0}(0)\right)^{T}
\end{array}\right]\left[\begin{array}{l}
\varepsilon \\
\varepsilon
\end{array}\right]
$$

Since path flows are unique for the restricted network, it is a simple matter to find arc flows from Eq. (28).

\subsection{The Cho-Smith-Friesz Alternative Derivation of Sensitivity Analysis Formulae}

The TFM is based on the implicit function theorem and requires local uniqueness. Specifically, we need to choose a unique path flow vector $h^{*}$ with a restricted network by solving the linear program (16). To meet the requirement of a nondegenerate extreme point, the number of paths with positive flow must equal the rank of $\left[\Delta^{T}, \Lambda^{T}\right]$. For convenience, we refer to that property as the positive-flow-rank (PFR) condition. It is sometimes not possible to fulfill the PFR condition even when working with the restricted network formulation of a given user equilibrium problem. Moreover, Bell and Iida (1997) observed the potential noninvertibility of the submatrix $\nabla_{h} C^{0}\left(h^{*}, 0\right)^{-1}$ of the Jacobian matrix $J_{h^{0} \mu^{0}}^{*}(0)$ employed by the TFM. Accordingly, Cho et al. (2000) proposed an arc-based reduction method that derives sensitivity analysis formulae without invoking the PFR condition.

In the arc-based reduction method, a "minimum distance" technique is employed to find a unique equilibrium path flow. To employ that perspective, a maximal set of rows from $\Delta$, say $\Delta_{1}$, is selected so that the combined matrix $\left[\Delta_{1}, \Lambda\right]^{T}$ is of full row rank. Thereby we achieve the following partition of $\Delta$ :

$$
\Delta=\left[\begin{array}{ll}
\Delta_{1} & \Delta_{2}
\end{array}\right]^{T}
$$

Therefore, there must exist matrices $M_{1}$ and $M_{2}$ such that

$$
\Delta_{2}=M_{1} \Delta_{1}+M_{2} \Lambda
$$

In concert, the arc-flow vector is partitioned so that

$$
v=\left[\begin{array}{ll}
v_{1} & v_{2}
\end{array}\right]^{T}
$$

where

$$
\begin{aligned}
& v_{2}=\Delta_{2} h \\
& =M_{1} \Delta_{1} h+M_{2} \Lambda h \\
& =M_{1} v_{1}+M_{2} T(\varepsilon)
\end{aligned}
$$

Since travel demands are linearly expressible in terms of arc flows, the following minimum norm projection problem may be employed to determine a unique equilibrium path-flow vector for any $v_{1}, \varepsilon$ and $h_{0}$. That is, we solve

$$
\min \left\|h-h_{0}\right\|^{2}=\left(h-h_{0}\right)^{T}\left(h-h_{0}\right) \text { s.t. }\left[\begin{array}{l}
\Delta_{1} \\
\Lambda
\end{array}\right] h=\left[\begin{array}{l}
v_{1} \\
T(\varepsilon)
\end{array}\right]
$$


Since the matrix $\left[\Delta_{1}, \Lambda\right]^{T}$ is of full row rank, there exists a unique solution $h\left(\varepsilon, v_{1}, h_{0}\right)$ to the above linearly constrained quadratic program, namely

$$
h\left(\varepsilon, v_{1}, h_{0}\right)=h_{0}+\left[\begin{array}{l}
\Delta_{1} \\
\Lambda
\end{array}\right]^{T}\left[\begin{array}{cc}
\Delta_{1} \Delta_{1}^{T} & \Delta_{1} \Lambda^{T} \\
\Lambda \Delta_{1}^{T} & \Lambda \Lambda^{T}
\end{array}\right]^{-1}\left(\left[\begin{array}{l}
v_{1} \\
T(\varepsilon)
\end{array}\right]-\left[\begin{array}{l}
\Delta_{1} \\
\Lambda
\end{array}\right] h_{0}\right)
$$

Moreover, if we let

$$
\left[\begin{array}{cc}
\Delta_{1} \Delta_{1}^{T} & \Delta_{1} \Lambda^{T} \\
\Lambda \Delta_{1}^{T} & \Lambda \Lambda^{T}
\end{array}\right]^{-1}=\left[\begin{array}{ll}
M_{11} & M_{12} \\
M_{21} & M_{22}
\end{array}\right]
$$

we can rewrite Eq. (34) in a compact form as

$$
h\left(\varepsilon, v_{1}, h_{0}\right)=N_{0} h_{0}+N_{1} v_{1}+N_{2} T(\varepsilon)
$$

where

$$
\begin{gathered}
N_{0}=\left[\begin{array}{cc}
I & 0 \\
0 & I
\end{array}\right]-\left[\begin{array}{c}
\Delta_{1} \\
\Lambda
\end{array}\right]^{T}\left[\begin{array}{cc}
\Delta_{1} \Delta_{1}^{T} & \Delta_{1} \Lambda^{T} \\
\Lambda \Delta_{1}^{T} & \Lambda \Lambda^{T}
\end{array}\right]^{-1}\left[\begin{array}{l}
\Delta_{1} \\
\Lambda
\end{array}\right] \\
N_{1}=\Delta_{1}^{T} M_{11}+\Lambda^{T} M_{21} \\
N_{2}=\Delta_{1}^{T} M_{12}+\Lambda^{T} M_{22}
\end{gathered}
$$

Also, from Eqs. (29) and, (35-37), $M_{1}$ and $M_{2}$ may be expressed as

$$
\Delta_{2}=M_{1} \Delta_{1}+M_{2} \Lambda=\left[\begin{array}{ll}
M_{1} & M_{2}
\end{array}\right]\left[\begin{array}{l}
\Delta_{1} \\
\Lambda
\end{array}\right]
$$

so that

$$
\begin{aligned}
{\left[\begin{array}{ll}
M_{1} & M_{2}
\end{array}\right] } & =\Delta_{2}\left[\begin{array}{l}
\Delta_{1} \\
\Lambda
\end{array}\right]^{T}\left(\left[\begin{array}{l}
\Delta_{1} \\
\Lambda
\end{array}\right]\left[\begin{array}{l}
\Delta_{1} \\
\Lambda
\end{array}\right]^{T}\right)^{-1} \\
& =\Delta_{2}\left[\begin{array}{l}
\Delta_{1} \\
\Lambda
\end{array}\right]^{T}\left[\begin{array}{ll}
M_{11} & M_{12} \\
M_{21} & M_{22}
\end{array}\right]=\Delta_{2}\left[\begin{array}{ll}
N_{1} & N_{2}
\end{array}\right]
\end{aligned}
$$

Therefore, $M_{1}=\Delta_{2} N_{1}$ and $M_{2}=\Delta_{2} N_{2}$. Finally, we conclude that the flowconservation conditions in Eq. (30) can be written as

$$
\Delta_{2} N_{1} v_{1}-v_{2}+\Delta_{2} N_{2} T(\varepsilon)=0
$$

so that

$$
M v+\Delta_{2} N_{2} T(\varepsilon)=0
$$

where

$$
M=\left[\begin{array}{ll}
\Delta_{2} N_{1} & -I
\end{array}\right]
$$


Carrying out the necessary algebra yield the following:

$$
\left[\begin{array}{c}
\nabla_{\varepsilon} v(0) \\
\nabla_{\varepsilon} \mu(0)
\end{array}\right]=\left[\begin{array}{cc}
\nabla_{\nu} c(v(0), 0) & -M^{T} \\
M^{T} & 0
\end{array}\right]^{-1}\left[\begin{array}{c}
-\nabla_{\varepsilon} c(v(0), 0) \\
\Delta_{2} N_{2} \nabla_{\varepsilon} Q(0)
\end{array}\right]
$$

from which it is apparent that differentiability of arc costs with respect to flow and parameter perturbations is needed; differentiability of demands with respect to parameter perturbations is also needed.

\section{Counterexamples in the Literature}

In this section we discuss some numerical examples appearing in the literature on user equilibrium sensitivity analysis.

\subsection{Counterexamples in Patriksson (2004), Josefsson and Patriksson (2007)} and Marcotte and Patriksson (2007)

In Patriksson (2004), there is an illustrative example explaining that the TFM can provide an inaccurate result. Also, Josefsson and Patriksson (2007) proposed three counterexamples which depicted some pitfalls of the TFM and which were re-used in Marcotte and Patriksson (2007). However, in the aforementioned examples, the authors either ignored the requirements of the TFM or applied the TFM to non-differentiable examples which violated the method's basic assumptions. As such the examples are not bonafide counter examples. In the discussion below, we will carry out TFM calculations for some of the aforementioned examples while enforcing the regularity conditions presented in Section 3 of this paper.

\subsubsection{Example 5 in Patriksson (2004)}

In Patriksson (2004), a 5 node, 7 arc network with 2 origin-destination (OD) pairs and 6 paths is depicted and repeated here as Fig. 1. There are two fixed travel demands for OD pairs $(1,4)$, and $(3,5)$. There are three paths corresponding to each OD pair: $p_{1}=\{1,3\}, p_{2}=\{1,7,4\}$ and $p_{3}=\{2,4\}$ for OD pair $(1,4)$ and $p_{4}=\{5,2\}, p_{5}=\{5,1,7\}$ and $p_{6}=\{6,7\}$ for OD pair $(3,5)$. The arc cost functions are

$$
\begin{gathered}
c_{1}\left(v_{1}\right)=10 v_{1} \\
c_{2}\left(v_{2}\right)=0.5 v_{2} \\
c_{3}\left(v_{3}\right)=3+10 v_{3} \\
c_{4}\left(v_{4}\right)=1+10 v_{4} \\
c_{5}\left(v_{5}\right)=v_{5} \\
c_{6}\left(v_{6}\right)=2+v_{6} \\
c_{7}\left(v_{7}\right)=v_{7}
\end{gathered}
$$

The travel demands are subject to perturbations expressed as the following vector:

$$
\varepsilon=\left[\begin{array}{ll}
\varepsilon_{14} & \varepsilon_{35}
\end{array}\right]^{T}
$$

When $\varepsilon=0$, the equilibrium arc flow solution is $v^{*}(0)=[0,2,0,1,1,0,0]^{T}$. However, as mentioned in Patriksson (2004), the solution does not obey strict complementarity. 


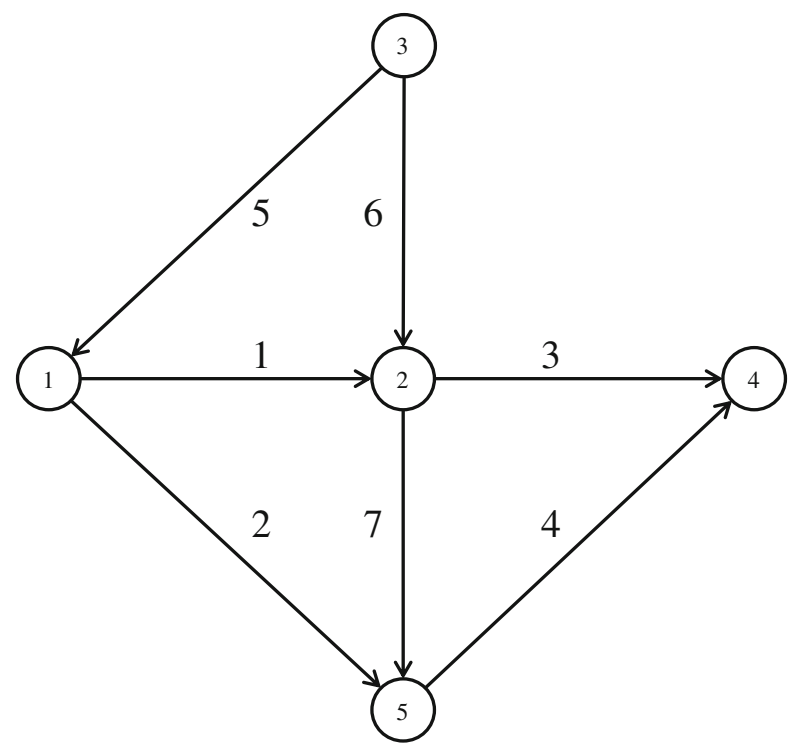

Fig. 1 The network of Patriksson's example 5

Also, the equilibrium solution is not differentiable. Therefore, this example problem is not one for which the TFM is applicable. That is, the TFM was never intended to apply to such a problem, and the example is not a counterexample.

\subsubsection{Example 7.3.1 in Josefsson and Patriksson (2007)}

This example, proposed by Josefsson and Patriksson (2007), considers the 4 node, 5 arc network with 2 OD pairs and 4 paths depicted in Fig. 2. There are fixed demands of 2 and 1 units of flow for OD pairs $(1,2)$ and $(4,2)$, respectively. There are four paths corresponding to the two OD pairs: $p_{1}=\{1\}, p_{2}=\{2,3\}, p_{3}=\{4\}$ and $p_{4}=\{5,3\}$. The arc cost functions are

$$
\begin{gathered}
c_{1}\left(v_{1}, \varepsilon\right)=2 v_{1}+\varepsilon \\
c_{2}\left(v_{2}\right)=v_{2} \\
c_{3}\left(v_{3}\right)=1 \\
c_{4}\left(v_{4}\right)=v_{4}+2 \\
c_{5}\left(v_{5}\right)=v_{5}
\end{gathered}
$$

When $\varepsilon=0$, the equilibrium arc flow solution is $v^{*}(0)=[1,1,2,0,1]^{T}$. (Note that the arc flow solution, namely $v^{*}(0)=[1,1,1,1,1]^{T}$, given in Josefsson and Patriksson (2007) is incorrect.) Since the solution violates strict complementary, the TFM should was never intended for application to this problem. However, since strict complementarity is not a necessary condition for differentiability, it is possible that the problem is differentiable in the neighborhood of $\varepsilon=0$ and that the TFM may be applied, as is next discussed.

We consider perturbations of the travel demands; that is, $\varepsilon=\left[\begin{array}{ll}\varepsilon_{12} & \varepsilon_{42}\end{array}\right]^{T}$. The unperturbed solution is $v^{*}(0)=[1,1,2,0,1]^{T}$, and the restricted arc-path and OD-path incidence matrices are 


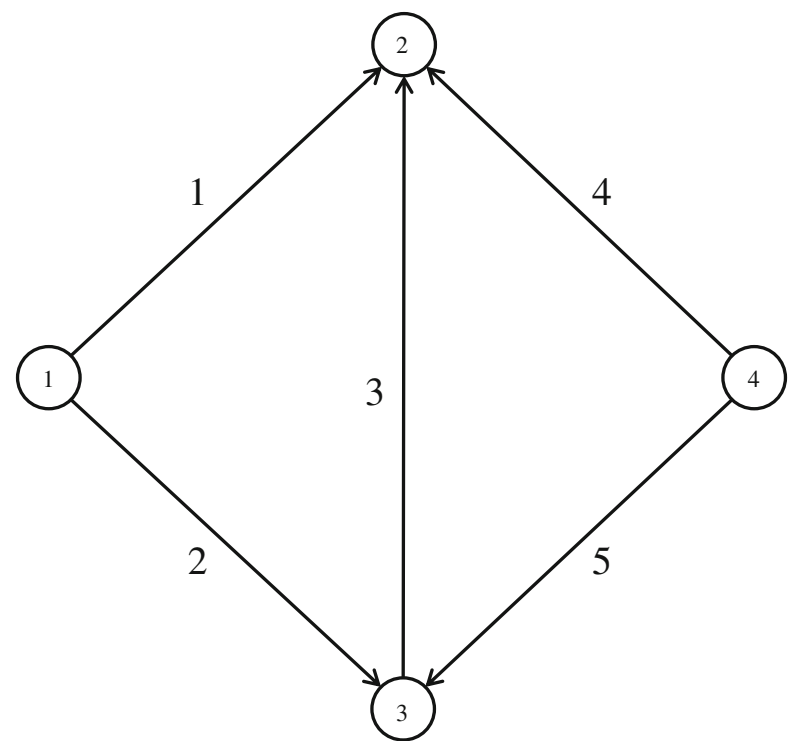

Fig. 2 The network of Josefsson and Patriksson's example 7.3.1

$$
\Delta=\left[\begin{array}{lll}
1 & 0 & 0 \\
0 & 1 & 0 \\
0 & 1 & 1 \\
0 & 0 & 1
\end{array}\right] \text { and } \Lambda=\left[\begin{array}{lll}
1 & 1 & 0 \\
0 & 0 & 1
\end{array}\right]
$$

In the restricted arc-path and OD-path incidence matrices, arc 4 is eliminated in the restricted network. The rows of the restricted arc-path incidence matrix correspond to arcs 1, 2, 3 and 5. The columns of arc-path incidence matrix correspond to paths 1,2 and 4, respectively. We solve a linear program to find non-degenerate extreme points of the restricted network with $b=\left[\begin{array}{lll}1 & 1 & 1\end{array}\right]^{T}$, and obtain the path flow solution $h^{0}(0)=\left[\begin{array}{lll}1 & 1 & 1\end{array}\right]^{T}>0$. The rank of $\left[\Delta^{0 T}, \Lambda^{0 T}\right]$ is equal to the number of paths with positive flow, which means that $h^{0}(0)$ is a non-degenerate extreme point. The scalar path flows corresponding to $h^{*}(0)$ are all positive, and $h^{0}, \Delta^{0}$ and $\Lambda^{0}$ are identical to $h^{*}, \Delta$ and $\Lambda$, respectively. It follows that

$$
\begin{aligned}
& {\left[\begin{array}{cc}
\nabla_{h} C^{0}\left(h^{*}, 0\right) & -\Lambda^{0 T} \\
\Lambda^{0} & 0
\end{array}\right]=\left[\begin{array}{ccccc}
2 & 0 & 0 & -1 & 0 \\
0 & 1 & 0 & -1 & 0 \\
0 & 0 & 1 & 0 & -1 \\
1 & 1 & 0 & 0 & 0 \\
0 & 0 & 1 & 0 & 0
\end{array}\right]} \\
& {\left[\begin{array}{c}
-\nabla_{\varepsilon} C^{0}\left(h^{*}, 0\right) \\
\nabla_{\varepsilon} Q(0)
\end{array}\right]=\left[\begin{array}{c}
-1 \\
0 \\
0 \\
0 \\
0
\end{array}\right]}
\end{aligned}
$$


Therefore

$$
\nabla_{\varepsilon} v^{*}=\left[\begin{array}{c}
-1 / 3 \\
1 / 3 \\
1 / 3 \\
0
\end{array}\right]
$$

which is identical to the solution reported in Josefsson and Patriksson (2007). We do add, however, that every cases for which strict complementarity condition will possess the differentiability that allowed the successful application of the TFM. However, the above calculations do show that sometimes violation of strict complementarity does not prevent application of the TFM.

\subsubsection{Example 7.3.2 in Josefsson and Patriksson (2007)}

In another example, Josefsson and Patriksson (2007) considered a 3 node, 4 arc network with the single OD pair $(1,3)$ and 4 paths, as depicted in Fig. 3. There is a fixed demand of 2 units of flow for OD pair $(1,3)$. Also there are four paths: $p_{1}=\{1,3\}$, $p_{2}=\{1,4\}, p_{3}=\{2,3\}$ and $p_{4}=\{2,4\}$. Furthermore, the arc cost functions are

$$
\begin{gathered}
c_{1}\left(v_{1}, \varepsilon\right)=v_{1}+\varepsilon \\
c_{2}\left(v_{2}\right)=v_{2} \\
c_{3}\left(v_{3}\right)=v_{3} \\
c_{4}\left(v_{4}\right)=v_{4}
\end{gathered}
$$

where $\varepsilon \in \mathfrak{R}^{1}$ is a now a scalar perturbation parameter. When $\varepsilon=0$, the equilibrium arc flow solution is $v^{*}(0)=[1,1,1,1]^{T}$. Thus, the restricted arc-path and OD-path incidence matrices are

$$
\Delta=\left[\begin{array}{llll}
1 & 1 & 0 & 0 \\
0 & 0 & 1 & 1 \\
1 & 0 & 1 & 0 \\
0 & 1 & 0 & 1
\end{array}\right] \text { and } \Lambda=\left[\begin{array}{llll}
1 & 1 & 1 & 1
\end{array}\right]
$$

In this example, the rank of $\left[\Delta^{T}, \Lambda^{T}\right]$ is 3 . As the analysis in Josefsson and Patriksson (2007) establishes, the possible number of paths having non-zero flow is either 2 or 4 . It is impossible to find a vector $b$ for which the linear program (16) yields is a nondegenerate path flow solution with only 3 non-zero flows. In other words, it is

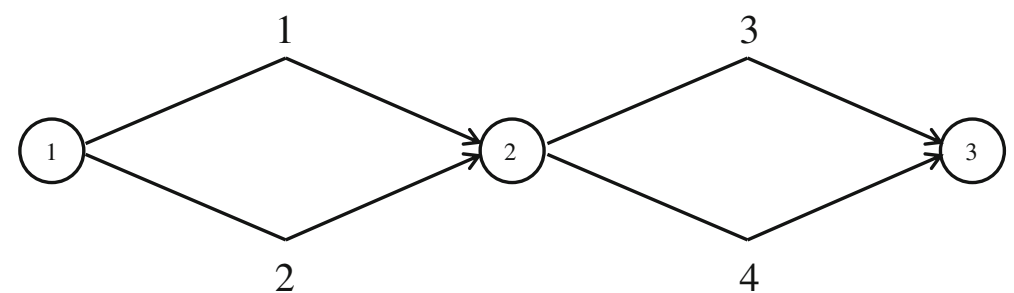

Fig. 3 The network of Josefsson and Patriksson's example 7.3.2 
impossible to satisfy the requirements of the TFM in this example. Therefore, the TFM should not be applied in this example to calculate the sensitivity information even though the gradient exists, and the example does not constitute a counterexample.

\subsubsection{Example 7.3.3 in Josefsson and Patriksson (2007)}

In this example, Josefsson and Patriksson (2007) considered the 3 node, 3 arc network with 3 OD pairs and 4 paths depicted in Fig. 4. There are three fixed demands of 1 unit of flow for each of the OD pairs $(1,2),(1,3)$ and $(3,2)$. There are four paths corresponding to the three OD pairs denoted by $p_{1}=\{1\}, p_{2}=\{2,3\}, p_{3}=\{2\}$ and $p_{4}=\{3\}$. The arc cost functions are given by

$$
\begin{gathered}
c_{1}\left(v_{1}, \varepsilon\right)=2 v_{1}+\varepsilon \\
c_{2}\left(v_{2}\right)=v_{2} \\
c_{3}\left(v_{3}\right)=v_{3}
\end{gathered}
$$

where $\varepsilon \in \mathfrak{R}^{1}$ is again a scalar perturbation parameter. When $\varepsilon=0$, the equilibrium arc flow solution is $v^{*}(0)=[1,1,1]^{T}$. Thus, the restricted arc-path and OD-path incidence matrices are

$$
\Delta=\left[\begin{array}{llll}
1 & 0 & 0 & 0 \\
0 & 1 & 1 & 0 \\
0 & 1 & 0 & 1
\end{array}\right] \text { and } \Lambda=\left[\begin{array}{llll}
1 & 1 & 0 & 0 \\
0 & 0 & 1 & 0 \\
0 & 0 & 0 & 1
\end{array}\right]
$$

In this example, the rank of $\left[\Delta^{T}, \Lambda^{T}\right]$ is 4 . Path 3 is the only path connecting OD pair (1, 3 ); so the flow on path 3 is exactly equal to the associated demand. Similarly, the flow on path 4 is exactly equal to the demand between $\operatorname{OD}$ pair $(3,2)$. Therefore, the flow on path 1 must be 1 and the flow on path 2 is zero. The flows just described form the unique equilibrium path flow solution, and the rank of $\left[\Delta^{T}, \Lambda^{T}\right]$ cannot possibly equal the number of paths with positive flow. Once again, this example does not satisfy the regularity conditions for the TFM. In addition, this example is non-differentiable, which fact also violates the TFM regularity conditions. Thus, the TFM should never be considered for application to this example, and the example, again, does not constitute a counterexample.

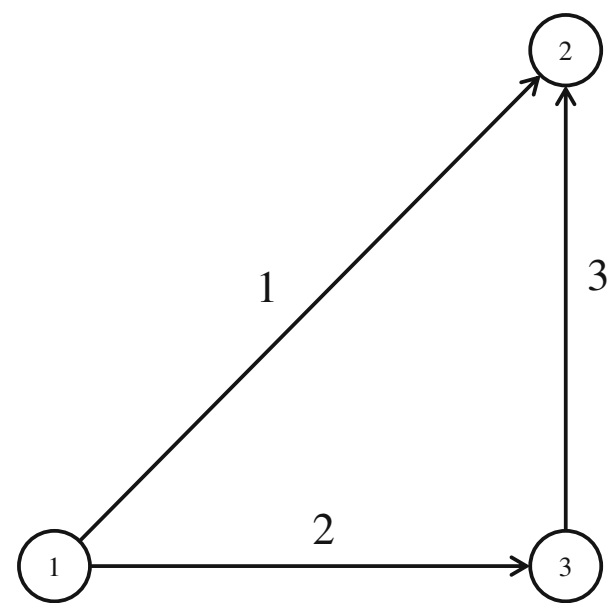

Fig. 4 The network of Josefsson and Patriksson's example 7.3.3 


\subsubsection{Modifying Example Problems to Satisfy the TFM Regularity Conditions}

Example 7.3.2 in Josefsson and Patriksson (2007), first discussed in this paper in Section 4.1.3 above, violates the regularity assumptions on which the TFM is predicated due to its symmetric arc cost functions and network topology. If we make the cost functions asymmetric, as we shall illustrate, the possible number of paths having non-zero flow is no longer restricted to either 2 or 4 . As depicted in Fig. 3. the network contains three nodes, four arcs, one OD pair and four paths. This example is identical to example 7.3.2 in Josefsson and Patriksson (2007) except for the cost function of arc 2 , which is now $c_{2}\left(v_{2}\right)=1+v_{2}$.

When $\varepsilon=0$, the equilibrium arc flow solution is $v^{*}(0)=[3 / 2,1 / 2,1,1]^{T}$. Thus, the restricted arc-path and OD-path incidence matrices are

$$
\Delta=\left[\begin{array}{llll}
1 & 1 & 0 & 0 \\
0 & 0 & 1 & 1 \\
1 & 0 & 1 & 0 \\
0 & 1 & 0 & 1
\end{array}\right] \text { and } \Lambda=\left[\begin{array}{llll}
1 & 1 & 1 & 1
\end{array}\right] .
$$

After solving the linear program (16), a path flow solution $h^{*}(0)=[1,1 / 2,0,1 / 2]^{T}$ can be obtained with $b=[1,1,1,1]^{T}$. In this case, the rank of $\left[\Delta^{T}, \Lambda^{T}\right]$ is equal to the number of paths with positive flow, which implies that $h^{*}$ is a non-degenerate extreme point. Due to the flow on path 3 being zero, we may eliminate path 3 to generate $h^{0 *}(0)=[1$, $1 / 2,1 / 2]^{T}$ which contains only those path variables having positive path flows. Conformally defined with respect to $h^{0^{*}}$, the reduced arc-path and OD-path incidence matrices, denoted by $\Delta^{0}$ and $\Lambda^{0}$ respectively, are

$$
\Delta^{0}=\left[\begin{array}{lll}
1 & 1 & 0 \\
0 & 0 & 1 \\
1 & 0 & 0 \\
0 & 1 & 1
\end{array}\right] \text { and } \Lambda^{0}=\left[\begin{array}{lll}
1 & 1 & 1
\end{array}\right] .
$$

It follows that

$$
\begin{gathered}
{\left[\begin{array}{cc}
\nabla_{h} C^{0}\left(h^{*}, 0\right) & -\Lambda^{0 T} \\
\Lambda^{0} & 0
\end{array}\right]=\left[\begin{array}{cccc}
2 & 1 & 0 & -1 \\
1 & 2 & 1 & -1 \\
0 & 1 & 2 & -1 \\
1 & 1 & 1 & 0
\end{array}\right]} \\
{\left[\begin{array}{l}
-\nabla_{\varepsilon} C^{0}\left(h^{*}, 0\right) \\
\nabla_{\varepsilon} Q(0)
\end{array}\right]=\left[\begin{array}{c}
-1 \\
-1 \\
0 \\
0
\end{array}\right]}
\end{gathered}
$$

Therefore, we have the following derivative of arc flows with respect to the perturbation $\varepsilon$ (of the cost function for arc 1):

$$
\nabla_{\varepsilon} v^{*}=\left[\begin{array}{c}
-1 / 2 \\
1 / 2 \\
0 \\
0
\end{array}\right]
$$


Clearly, all requirements of the TFM are satisfied and the derivative of differentiable solutions with respect to perturbation parameters can be calculated by the TFM. This contrasts to the example 7.3.3 in Josefsson and Patriksson (2007) and the example 5 in Patriksson (2004), for which nondifferentiability is encountered in violation of the assumptions intrinsic to the TFM.

\subsubsection{Applying Cho-Smith-Friesz Method on Counterexamples}

We illustrate in this section that the Cho-Smith-Friesz method (CSFM) extends the circumstances under which the TFM is applicable.

Example 7.3.2 in Josefsson and Patriksson (2007) This example has been stated previously. Recall that, when $\varepsilon=0$, the equilibrium arc flow solution is $v^{*}(0)=[1,1,1,1]^{T}$ and the restricted arc-path and OD-path incidence matrices are

$$
\Delta=\left[\begin{array}{llll}
1 & 1 & 0 & 0 \\
0 & 0 & 1 & 1 \\
1 & 0 & 1 & 0 \\
0 & 1 & 0 & 1
\end{array}\right] \text { and } \Lambda=\left[\begin{array}{llll}
1 & 1 & 1 & 1
\end{array}\right]
$$

In this example, the rank of $\left[\Delta^{T}, \Lambda^{T}\right]$ is 3 . By inspection, $\Lambda$ has one independent row. Also the rows of $\Delta$ that correspond to arcs 1 and 3 are linearly independent, so that we may partition $\Delta$ according to

$$
\Delta=\left[\begin{array}{ll}
\Delta_{1} & \Delta_{2}
\end{array}\right]^{T}
$$

where

$$
\Delta_{1}=\left[\begin{array}{llll}
1 & 1 & 0 & 0 \\
1 & 0 & 1 & 0
\end{array}\right] \text { and } \Delta_{2}=\left[\begin{array}{llll}
0 & 0 & 1 & 1 \\
0 & 1 & 0 & 1
\end{array}\right]
$$

The matrices referred to in Eq. (41) are the followings:

$$
\begin{gathered}
\nabla_{\nu} c(v(0), 0)=\left[\begin{array}{llll}
1 & 0 & 0 & 0 \\
0 & 1 & 0 & 0 \\
0 & 0 & 1 & 0 \\
0 & 0 & 0 & 1
\end{array}\right] \\
M=\left[\begin{array}{cccc}
-1 & -1 & 0 & 0 \\
0 & 0 & -1 & -1
\end{array}\right] \\
-\nabla_{\varepsilon} c(v(0), 0)=\left[\begin{array}{l}
1 \\
0 \\
0 \\
0
\end{array}\right] \\
\Delta_{2} N_{2} \nabla_{\varepsilon} Q(0)=\left[\begin{array}{l}
0 \\
0
\end{array}\right]
\end{gathered}
$$


Therefore, Eq. (41) reduces to the following:

$$
\nabla_{\varepsilon} v^{*}=\left[\begin{array}{c}
-1 / 2 \\
1 / 2 \\
0 \\
0
\end{array}\right]
$$

which is identical to the solution in Josefsson and Patriksson (2007). Thereby, we see that the CSFM may sometimes be able to deal with cases wherein $h^{*}$ is not a nondegenerate path solution.

Example 7.3.3 in Josefsson and Patriksson (2007) When $\varepsilon=0$, the equilibrium arc flow solution is $v^{*}(0)=[1,1,1]^{T}$. Thus, the restricted arc-path and OD-path incidence matrices are

$$
\Delta=\left[\begin{array}{llll}
1 & 0 & 0 & 0 \\
0 & 1 & 1 & 0 \\
0 & 1 & 0 & 1
\end{array}\right] \text { and } \Lambda=\left[\begin{array}{llll}
1 & 1 & 0 & 0 \\
0 & 0 & 1 & 0 \\
0 & 0 & 0 & 1
\end{array}\right]
$$

In this example, the rank of $\left[\Delta^{T}, \Lambda^{T}\right]$ is 3 . By inspection $\Lambda$ has three independent rows. Moreover, the rows of $\Delta$ are linearly independent. Thus

$$
\Delta=\Delta_{2}
$$

based on the notation introduced in Eq. (42). Therefore, the matrices of Eq. (41) may be expressed as

$$
\begin{gathered}
\nabla_{\nu} c(v(0), 0)=\left[\begin{array}{lll}
2 & 0 & 0 \\
0 & 1 & 0 \\
0 & 0 & 1
\end{array}\right] \\
M=\left[\begin{array}{ccc}
-1 & 0 & 0 \\
0 & -1 & 0 \\
0 & 0 & -1
\end{array}\right] \\
-\nabla_{\varepsilon} c(v(0), 0)=\left[\begin{array}{c}
-1 \\
0 \\
0
\end{array}\right] \\
\Delta_{2} N_{2} \nabla_{\varepsilon} Q(0)=\left[\begin{array}{l}
0 \\
0 \\
0
\end{array}\right]
\end{gathered}
$$

Furthermore, Eq. (41) yields

$$
\nabla_{\varepsilon} v^{*}=\left[\begin{array}{l}
0 \\
0 \\
0
\end{array}\right]
$$

which is the directional derivative when $\varepsilon=1$ employed in the Josefsson-Patriksson solution. However, the directional derivative when $\varepsilon=-1$ cannot be obtained by the CSFM because it corresponds to a non-differentiable circumstance, and thereby violates the regularity conditions needed for application of the CSFM method. 
Subsequent to Bell and Iida (1997), non-invertibility of the Jacobian of the path cost occurring in Eq. (24) of the TFM was also observed by Yang and Bell (2007). The Jacobian of the path cost is in general not invertible so that the sensitivity formulae of the TFM would seem to fail in such a circumstance. However, Yang and Bell (2007) presented an example for which the Jacobian of the path cost is not invertible yet the requisite information for sensitivity analysis exists. As we have already indicated, this is not a surprise, since the invertibility of the Jacobian of path cost is a regularity condition for the TFM that one may relax when it is realized that the sensitivity analysis formulae may be derived via the CSFM. More generally, the TFM sensitivity formulae remain applicable when the Jacobian of the path cost is not invertible. This understanding, as we now reiterate, is established by the analysis of Cho et al. (2000) and the summary thereof presented in Section 3.3 of this paper, because Cho et al. (2000) derive those formulae without reference to the network's topology or the presumption of invertibility of the Jacobian of path cost. We note that the Yang and Bell (2007) counter example is used in Yang and Bell (2007); thus, the above remarks also apply to the Yang and Huang (2005) paper. Our remarks immediately above are not criticisms of the manuscripts cited but rather are meant to establish connections among the various papers on the subject of equilibrium sensitivity analysis that have appeared over a considerable period of time in various journals and books.

\subsubsection{Example 6 in Yang and Bell (2007)}

In this example, Yang and Bell (2007) considered a 3 node, 2 arc network with 3 OD pairs and 3 paths which is depicted in Fig. 5. There are two fixed demands of 5 units of flow for each of the OD pairs $(1,2)$ and $(2,3)$. The demand for OD pair $(1,3)$ is perturbed and becomes $5+\varepsilon_{13}$. The three paths are $p_{1}=\{1\}, p_{2}=\{2\}$ and $p_{3}=\{1,2\}$. The arc cost functions are given by

$$
\begin{gathered}
c_{1}\left(v_{1}, \varepsilon_{1}\right)=v_{1}+1+\varepsilon_{1} \\
c_{2}\left(v_{2}\right)=v_{2}+1
\end{gathered}
$$

It is helpful to define

$$
\varepsilon=\left[\begin{array}{l}
\varepsilon_{13} \\
\varepsilon_{1}
\end{array}\right] .
$$

When $\varepsilon=0$, the equilibrium arc flow solution is $v^{*}(0)=[10,10]^{T}$. Thus, the restricted arc-path and OD-path incidence matrices are

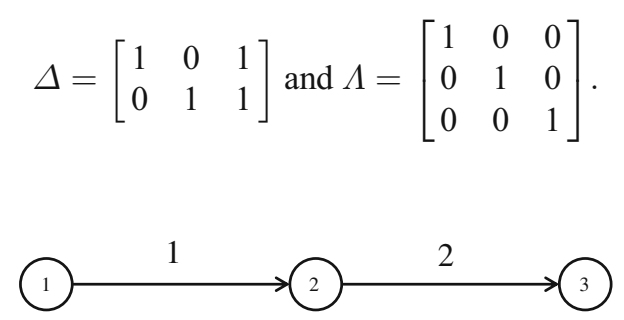

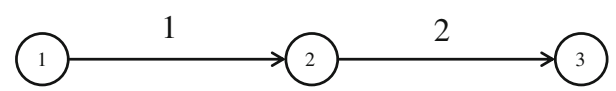

Fig. 5 The network of Yang and Bell's example 6 
Solving the linear programming (16), with $b=[1,1,1]^{T}$, a path flow solution $h^{*}=[5,5,5]^{T}$ is obtained. The rank of $\left[\Delta^{T}, \Lambda^{T}\right]$ is equal to the number of paths with positive flow, which implies that $h^{*}$ are all positive. Moreover, $h^{0^{*}}, \Delta^{0^{*}}$ and $\Lambda^{0^{*}}$ are identical to $h^{*}, \Delta$ and $\Lambda$, respectively. By inspection, we have

$$
\left[\begin{array}{cc}
\nabla_{h} C^{0}\left(h^{*}, 0\right) & -\Lambda^{0 T} \\
\Lambda^{0} & 0
\end{array}\right]=\left[\begin{array}{cccccc}
1 & 0 & 1 & -1 & 0 & 0 \\
0 & 1 & 1 & 0 & -1 & 0 \\
1 & 1 & 2 & 0 & 0 & -1 \\
1 & 0 & 0 & 0 & 0 & 0 \\
0 & 1 & 0 & 0 & 0 & 0 \\
0 & 0 & 1 & 0 & 0 & 0
\end{array}\right]
$$

Yang and Bell (2007) note that the inverse of the Jacobian of the path cost matrix,

$$
\nabla_{h} C^{0}\left(h^{*}, 0\right)=\left[\begin{array}{lll}
1 & 0 & 1 \\
0 & 1 & 1 \\
1 & 1 & 2
\end{array}\right]^{-1}
$$

does not exist, and it would seem that the TFM is not applicable. However, the inverse of the entire matrix presented on the right-hand side of Eq. (43) is easily shown to be the following:

$$
\left[\begin{array}{cc}
\nabla_{\varepsilon} C^{0}\left(h^{*}, 0\right) & -\Lambda^{0 T} \\
\Lambda^{0} & 0
\end{array}\right]^{-1}=\left[\begin{array}{cccccc}
0 & 0 & 0 & 1 & 0 & 0 \\
0 & 0 & 0 & 0 & 1 & 0 \\
0 & 0 & 0 & 0 & 0 & 1 \\
-1 & 0 & 0 & 1 & 0 & 1 \\
0 & -1 & 0 & 0 & 1 & 1 \\
0 & 0 & -1 & 1 & 1 & 2
\end{array}\right]
$$

Also we note that

$$
\left[\begin{array}{c}
-\nabla_{\varepsilon} C^{0}\left(h^{*}, 0\right) \\
\nabla_{\varepsilon} Q(0)
\end{array}\right]=\left[\begin{array}{cc}
-1 & 0 \\
0 & 0 \\
-1 & 0 \\
0 & 0 \\
0 & 0 \\
0 & 1
\end{array}\right]
$$

According to Eqs. (17-18), the derivative of arc flows with respect to $\varepsilon$ is

$$
\nabla_{\varepsilon} v^{*}=\left[\begin{array}{ll}
0 & 1 \\
0 & 1
\end{array}\right]
$$

which is identical to the solution in Yang and Bell (2007). The derivative of equilibrium costs with respect to $\varepsilon$ is

$$
\nabla_{\varepsilon} \mu^{*}=\left[\begin{array}{ll}
1 & 1 \\
0 & 1 \\
1 & 2
\end{array}\right]
$$

which is also identical with the solution in Yang and Bell (2007). Thus, the TFM may remain applicable even though the Jacobian of the path cost matrix is not invertible. 
More precisely, the non-invertibility problem encountered in expression (44) need not be inherited by inverse matrix in Eq. (17).

\subsubsection{Example 8 in Yang and Bell (2007)}

In this example, Yang and Bell (2007) considered a 3 node, 4 arc network with 3 OD pairs and 8 paths. The network topology is that of Fig. 3. The OD demands are given by $d_{13}=2+\varepsilon_{13}, d_{12}=2+\varepsilon_{12}$ and $d_{23}=2+\varepsilon_{23}$. There are eight paths corresponding to three OD pairs; they are $h_{1}=\{1,3\}, h_{2}=\{1,4\}, h_{3}=\{2,3\}, h_{4}=\{2,4\}, h_{5}=\{1\}, h_{6}=\{2\}$, $h_{7}=\{3\}$ and $h_{8}=\{4\}$. The arc cost functions are given by

$$
\begin{aligned}
c_{1}\left(v_{1}, \varepsilon\right) & =v_{1}+1+\varepsilon \\
c_{2}\left(v_{2}\right) & =v_{2}+1 \\
c_{3}\left(v_{3}, \varepsilon\right) & =v_{3}+1+\varepsilon \\
c_{4}\left(v_{4}\right) & =v_{4}+1
\end{aligned}
$$

where $\varepsilon \in \mathfrak{R}^{1}$ is a now a scalar perturbation parameter. When $\varepsilon=0$, the equilibrium arc flow solution is $v^{*}(0)=[2,2,2,2]^{T}$ and the arc-path and OD-path incidence matrices are

$$
\Delta=\left[\begin{array}{llllllll}
1 & 1 & 0 & 0 & 1 & 0 & 0 & 0 \\
0 & 0 & 1 & 1 & 0 & 1 & 0 & 0 \\
1 & 0 & 1 & 0 & 0 & 0 & 1 & 0 \\
0 & 1 & 0 & 1 & 0 & 0 & 0 & 1
\end{array}\right] \text { and } \Lambda=\left[\begin{array}{llllllll}
1 & 1 & 1 & 1 & 0 & 0 & 0 & 0 \\
0 & 0 & 0 & 0 & 1 & 1 & 0 & 0 \\
0 & 0 & 0 & 0 & 0 & 0 & 1 & 1
\end{array}\right]
$$

In this example, the rank of $\left[\Delta^{T}, \Lambda^{T}\right]$ is 5 , but it appears to be impossible to find a vector $b$ such that the path flow solution of linear programming problem (16) having only five non-zero path flow variables. Therefore, the non-degeneracy condition cannot be satisfied, and it is inappropriate to employ the TFM for sensitivity analysis of the example considered in this section.

\subsubsection{Modifying Example Problems to Satisfy the Tobin and Friesz's Regularity Conditions}

In example 8 of Yang and Bell (2007), the number of paths is greater than the number of arcs plus the number of OD pairs. It can be hard to find a non-degenerate extreme point that satisfies the regularity conditions needed for the TFM. This observation is readily apparent when applying the TFM to example 8 of Yang and Bell (2007). In some cases, and this example is one of them, it is possible to modify the problem of interest to satisfy the missing regularity conditions. One way we can do that is reducing the number of paths needed to make the non-degenerate extreme point exist. If we eliminate OD pairs $(1,2)$ and $(2,3)$, this example will be a 3 node, 4 arc network with 1 OD pair and 4 paths, which is similar to the example 7.3.2 in Josefsson and Patriksson (2007).

In particular, there are four paths corresponding to OD pair $(1,3)$, denoted by $p_{1}=\{1,3\}, p_{2}=\{1,4\}, p_{3}=\{2,3\}$ and $p_{4}=\{2,4\}$. The arc cost functions are:

$$
\begin{aligned}
c_{1}\left(v_{1}, \varepsilon\right) & =v_{1}+1+\varepsilon \\
c_{2}\left(v_{2}\right) & =v_{2}+1 \\
c_{3}\left(v_{3}, \varepsilon\right) & =v_{3}+1+\varepsilon \\
c_{4}\left(v_{4}\right) & =v_{4}+1
\end{aligned}
$$


where $\varepsilon \in \mathfrak{R}^{1}$ is a now a scalar perturbation parameter. Moreover, the arc cost function and the network topology are symmetric, reminiscent of the example 7.3.2 in Josefsson and Patriksson (2007). In order to modify the counterexample to satisfy the requirement of the TFM, we may easily make the cost functions asymmetric to attain this goal. For simplicity, we modify the cost function of arc 2 to $c_{2}\left(v_{2}\right)=v_{2}$. When $\varepsilon=0$, the equilibrium arc flow solution is $v^{*}(0)=[1 / 2,3 / 2,1,1]^{T}$. Thus, the arc-path and ODpath incidence matrices are

$$
\Delta=\left[\begin{array}{llll}
1 & 1 & 0 & 0 \\
0 & 0 & 1 & 1 \\
1 & 0 & 1 & 0 \\
0 & 1 & 0 & 1
\end{array}\right] \text { and } \Lambda=\left[\begin{array}{llll}
1 & 1 & 1 & 1
\end{array}\right]
$$

The rank of $\left[\Delta^{T}, \Lambda^{T}\right]$ is 3 . Using $b=[1,1,1,1]^{T}$, the linear program (16) yields the path flow vector $h^{*}=[1 / 2,0,1 / 2,1]^{T}$. In that case, the rank of $\left[\Delta^{T}, \Lambda^{T}\right]$ is equal to the number of paths with positive flow, which implies that $h^{*}$ is a non-degenerate extreme point. Since the flow on path 2 is zero, we can eliminate path 2 to generate $h^{0^{*}}$ which contains only those path variables having positive path flows. Corresponding to $h^{0 *}$ we have the following reduced arc-path and OD-path incidence matrices:

$$
\Delta^{0}=\left[\begin{array}{lll}
1 & 0 & 0 \\
0 & 1 & 1 \\
1 & 1 & 0 \\
0 & 0 & 1
\end{array}\right] \text { and } \Lambda^{0}=\left[\begin{array}{lll}
1 & 1 & 1
\end{array}\right] .
$$

Accordingly, we have

$$
\begin{gathered}
{\left[\begin{array}{cc}
\nabla_{h} C^{0}\left(h^{*}, 0\right) & -\Lambda^{0 T} \\
\Lambda^{0} & 0
\end{array}\right]=\left[\begin{array}{cccc}
2 & 1 & 0 & -1 \\
1 & 2 & 1 & -1 \\
0 & 1 & 2 & -1 \\
1 & 1 & 1 & 0
\end{array}\right]} \\
{\left[\begin{array}{c}
-\nabla_{\varepsilon} C^{0}\left(h^{*}, 0\right) \\
\nabla_{\varepsilon} Q(0)
\end{array}\right]=\left[\begin{array}{ccc}
-1 & -1 & 0 \\
0 & -1 & 0 \\
0 & 0 & 0 \\
0 & 0 & 1
\end{array}\right]}
\end{gathered}
$$

and the derivative of arc flows with respect $\varepsilon_{1}, \varepsilon_{3}$ and $\varepsilon_{13}$ is

$$
\nabla_{\varepsilon} v^{*}=\left[\begin{array}{ccc}
-1 / 2 & 0 & 1 / 2 \\
1 / 2 & 0 & 1 / 2 \\
0 & -1 / 2 & 1 / 2 \\
0 & 1 / 2 & 1 / 2
\end{array}\right]
$$

In this modified example, all requirements of the TFM are satisfied. Therefore, the derivative of differentiable solutions with respect to perturbation parameters can be calculated using the TFM. Obviously the modified problem is significantly different than the original problem; as such, sensitivity analysis of the modified problem may be of little value. 


\subsubsection{Applying Cho-Smith-Friesz Method on Counterexamples}

Let us again consider example 8 in Yang and Bell (2007). When $\varepsilon=0$, the equilibrium arc flow solution is $v^{*}(0)=[2,2,2,2]^{T}$. Thus, the restricted arc-path and OD-path incidence matrices are

$$
\Delta=\left[\begin{array}{llllllll}
1 & 1 & 0 & 0 & 1 & 0 & 0 & 0 \\
0 & 0 & 1 & 1 & 0 & 1 & 0 & 0 \\
1 & 0 & 1 & 0 & 0 & 0 & 1 & 0 \\
0 & 1 & 0 & 1 & 0 & 0 & 0 & 1
\end{array}\right] \text { and } \Lambda=\left[\begin{array}{llllllll}
1 & 1 & 1 & 1 & 0 & 0 & 0 & 0 \\
0 & 0 & 0 & 0 & 1 & 1 & 0 & 0 \\
0 & 0 & 0 & 0 & 0 & 0 & 1 & 1
\end{array}\right]
$$

In this example, the rank of $\left[\Delta^{T}, \Lambda^{T}\right]$ is 5 . Clearly $\Lambda$ has three independent rows. Choosing two more linearly independent rows form $\Delta$, say those corresponding to arcs 1 and 3 , allows $\Delta$ to be partitioned in this fashion:

$$
\Delta=\left[\begin{array}{ll}
\Delta_{1} & \Delta_{2}
\end{array}\right]^{T}
$$

where

$$
\Delta_{1}=\left[\begin{array}{llllllll}
1 & 1 & 0 & 0 & 1 & 0 & 0 & 0 \\
1 & 0 & 1 & 0 & 0 & 0 & 1 & 0
\end{array}\right] \text { and } \Delta_{1}=\left[\begin{array}{llllllll}
0 & 0 & 1 & 1 & 0 & 1 & 0 & 0 \\
0 & 1 & 0 & 1 & 0 & 0 & 0 & 1
\end{array}\right] .
$$

The individual submatrices of matrix Eq. (41) are the following:

$$
\begin{gathered}
\nabla_{v} c(v(0), 0)=\left[\begin{array}{cccc}
1 & 0 & 0 & 0 \\
0 & 1 & 0 & 0 \\
0 & 0 & 1 & 0 \\
0 & 0 & 0 & 1
\end{array}\right] \\
M=\left[\begin{array}{cccc}
-1 & -1 & 0 & 0 \\
0 & 0 & -1 & -1
\end{array}\right] \\
-\nabla_{\varepsilon} c(v(0), 0)=\left[\begin{array}{ccccc}
1 & 0 & 0 & 0 & 0 \\
0 & 0 & 0 & 0 & 0 \\
0 & 1 & 0 & 0 & 0 \\
0 & 0 & 0 & 0 & 0
\end{array}\right] \\
\Delta_{2} N_{2} \nabla_{\varepsilon} Q(0)=\left[\begin{array}{ccccc}
0 & 0 & -1 & -1 & 0 \\
0 & 0 & -1 & 0 & -1
\end{array}\right]
\end{gathered}
$$

Therefore, Eq. (41) yields

$$
\nabla_{\varepsilon} v^{*}=\left[\begin{array}{ccccc}
-1 / 2 & 0 & 1 / 2 & 1 / 2 & 0 \\
1 / 2 & 0 & 1 / 2 & 1 / 2 & 0 \\
0 & -1 / 2 & 1 / 2 & 0 & 1 / 2 \\
0 & 1 / 2 & 1 / 2 & 0 & 1 / 2
\end{array}\right]
$$

In the matrix immediately above, columns 1 through 5 are the derivatives of arc flows with respect to the perturbations $\varepsilon_{1}, \varepsilon_{2}, \varepsilon_{12}, \varepsilon_{13}$ and $\varepsilon_{23}$, respectively. The resulting approximation of the CSFM is identical with Yang and Bell (2007). Thereby, it overcomes the non-invertibility problem noted by Bell and Iida (1997). As such the CSFM is more general than TFM in that it solves problems without requiring the PFR condition. 


\section{Conclusion}

This paper has reviewed several prior articles in which some defects of the Tobin-Friesz method (TFM) are indicated and some examples are shown to explain that the TFM may fail or lead to an incorrect solution. However, we found this is not because the TFM is incorrect but because some of the so-called counter examples were purposely fabricated to violate the assumptions intrinsic to the TFM and the related Cho-Smith-Friesz method (CSFM). That is, the counter examples reported in the literature are not true counter examples for the TFM because they violate the regularity conditions on which the method is based.

Nonetheless, we readily acknowledge that the TFM must be applied with care. The reward for exercising such care is the rather simple formulae that characterize the TFM. In this paper, we have seen that, sometimes when the regularity conditions of the TFM are violated, the equilibrium problem of interest may be modified to allow the method's application; this is especially so when cost symmetries are the complicating aspect of a given problem. We also observed that sometimes the Cho-Smith-Friesz method (CSFM) may be employed in lieu of the TFM to deal with noninvertible Jacobians.

\section{References}

Bell MGH, Iida Y (1997) Transportation network analysis. Wiley, New Jersey

Cho H, Smith T, Friesz TL (2000) A reduction method for local sensitivity analyses of network equilibrium arc flows. Transp Res Part B 34:31-51

Dafermos S (1988) Sensitivity analysis in variational inequalities. Math Oper Res 13:421-434

Davis G (1994) Exact local solution of the continuous network design problem via stochastic user equilibrium assignment. Transp Res Part B 28:61-75

Fiacco A (1983) Introduction to sensitivity and stability analysis in nonlinear programming. Academic, New York

Friesz TL, Tobin R, Cho H, Mehta N (1990) Sensitivity analysis based heuristic algorithms for mathematical programs with variational inequality constraints. Math Program 48:265-284

Josefsson M, Patriksson M (2007) Sensitivity analysis of separable traffic equilibrium equilibria with application to bilevel optimization in network design. Transp Res Part B 41:4-31

Kyparisis J (1987) Sensitivity analysis framework for variational inequalities. Math Program 38:203-213

Lu S (2008) Sensitivity of static traffic user equilibria with perturbations in arc cost function and travel demand. Transp Sci 42:105-123

Marcotte P, Patriksson M (2007) Handbooks in operations research and management science: transportation. In: Barnhart C, Laporte G (eds) Traffic equilibrium. Elsevier, Netherlands, pp 623-714

Patriksson M (2004) Sensitivity analysis of traffic equilibria. Transp Sci 38:258-281

Patriksson M, Rockafellar R (2003) Sensitivity analysis of aggregated variational inequality problems with application to traffic equilibria. Transp Sci 37:56-68

Qiu Y, Magnanti T (1989) Sensitivity analysis for variational inequalities defined on polyhedral sets. Math Oper Res 14:410-432

Robinson SM (2006) Strong regularity and the sensitivity analysis of traffic equilibria: a comment. Transp Sci 40:540-542

Tobin RL (1986) Sensitivity analysis for variational inequalities. J Optim Theory Appl 48:191-204

Tobin RL, Friesz TL (1988) Sensitivity analysis for equilibrium network flow. Transp Sci 22:242-250

Yang H (1997) Sensitivity analysis for the elastic-demand network equilibrium problem with applications. Transp Res Part B 31:55-70

Yang H, Bell M (2007) Sensitivity analysis of network traffic equilibria revisited: the corrected approach. In: Heydecker BG (ed) Mathematics in transport. Elsevier, Netherlands, pp 373-395

Yang H, Huang H (2005) Mathematical and economic theory of road pricing. Elsevier, Netherlands

Yang H, Meng Q, Bell M (2001) Simultaneous estimation of the origin-destination matrices and travel-cost coefficient for congested networks in a stochastic user equilibrium. Transp Sci 35:107-123

Ying J, Yang H (2005) Sensitivity analysis of stochastic user equilibrium flows in a bi-modal network with application to optimal pricing. Transp Res Part B 39:769-795 